\title{
Physics-Based Interpretation of Tool-Workpiece Interface Temperature Signals for Detection of Defect Formation during Friction Stir Welding
}

\author{
Amber Shrivastava, Clemens Dingler, Michael Zinn, Frank E. Pfefferkorn * \\ Department of Mechanical Engineering, University of Wisconsin-Madison, USA \\ *corresponding author \\ e-mail:pfefferk@engr.wisc.edu \\ phone: 001-608-263-2668
}

\begin{abstract}
The objective of the work is the reduction and eventual avoidance of post welding inspections that are currently needed to ensure defect-free welds. An analytical thermal model of the FSW process along with an analytical disturbance model is developed. This disturbance model relates defect formation to variations in the measured temperature and is based on experimental process identification. A dynamic disturbance observer computes an estimate of the disturbance signal, which is further processed in order to provide information about the presence of defects along the weld. Experiments for one kind of disturbance verify that the observer shows good tracking behavior.

\section{Introduction}

Friction stir welding (FSW) was invented at The Welding Institute (TWI) of UK in 1991 [1]. The advantages of Friction stir welding (FSW) are significant, including energy savings, superior joint mechanical properties, and lower process environmental impact as compared to other welding processes [2-4]. However, while FSW is an inherently cost-effective welding process, the need for significant weld inspection, particularly in the case of high-reliability applications, can increase the cost of FSW by a factor of three or more, making it cost-prohibitive. Therefore, a new approach to weld inspection is required - where characterization of weld quality can be obtained in real-time, drastically reducing the need for post-process inspection. The objective of this work is to create a FSW defect detection approach using physics-based process and defect dynamic modeling. The use of process and defect dynamic models to filter or condition the measured process outputs significantly improves the detection. Analytical thermal model, disturbance model and disturbance observer developed for this work are explained below.
\end{abstract}

\subsection{Thermal process model}

A physics-based thermal process model was developed that relates process inputs (i.e. tool rotation frequency, $f[\mathrm{~Hz}]$ and traverse speed, $v$ ) to the measured process output (i.e. tool-workpiece interface temperature). The thermal process model uses a transient, lumped-parameter approach. Figure 1 shows a sketch of the thermal model. For this model $m_{s / z}$ represents the mass of shear layer zone (SLZ) which rotates with the spinning tool, $T_{s / z}$ is the temperature of the SLZ, $\dot{m}_{i}$ and $\dot{m}_{o}$ are mass flow in and mass flow out rates, and $q_{g e n}, q_{a o}, q_{a i}$ and $q_{c}$ are heat generated due to plastic deformation in SLZ, heat advected out with mass flowing out, heat advected in with mass flowing in and heat conducted out to work piece and backing plate, respectively. It was assumed that: all the energy from the spindle is converted to thermal energy, i.e., $q_{g e n}=\tau \omega$, where $\tau$ is torque and $\omega$ is angular tool rotation speed; workpiece and backing plate temperatures are constant and equal to room temperature, $T_{R}$, i.e., $T_{W}=T_{B}=T_{R}$; no flash is generated during welding; convection and radiation heat losses to the surroundings are negligible; heat loss through the FSW tool is negligible; and thermo-physical properties are constant, uniform, and evaluated at a representative temperature of $400^{\circ} \mathrm{C}$. 


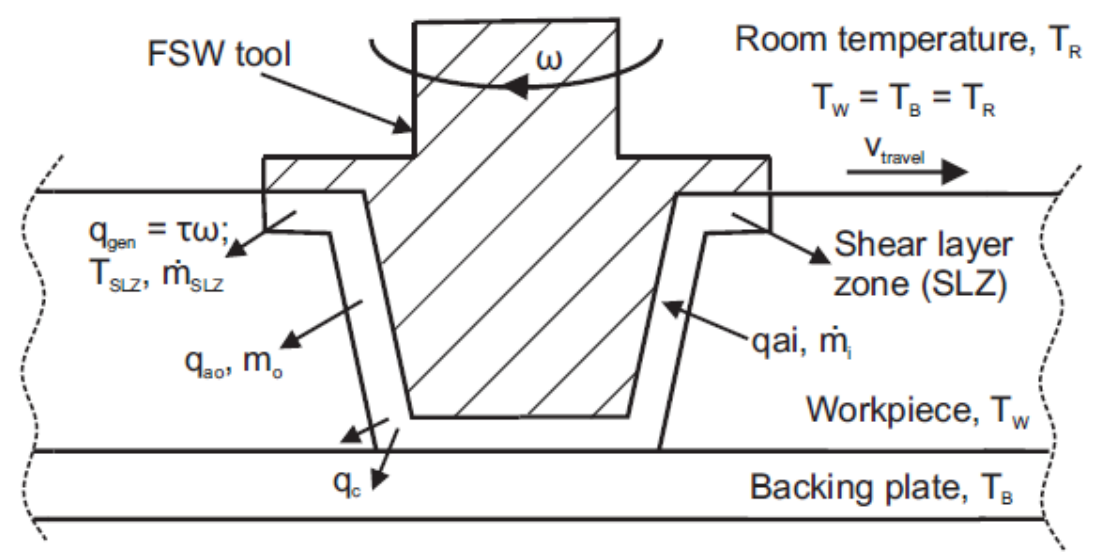

Figure 1. Schematic of the lumped-parameter thermal model

For this system, conservation of energy and mass for the SLZ was written as

$$
\begin{gathered}
m_{s l z} c_{s l z} \frac{d T_{s l z}}{d t}=q_{g e n}+q_{a i}-q_{a o}-q_{c} \\
\dot{m}_{i}-\dot{m}_{o}=\dot{m}_{s l z}
\end{gathered}
$$

Substituting $q_{g e n}=\tau \omega, q_{a i}=\dot{m}_{i} c_{w} T_{w}, q_{a o}=\dot{m}_{o} c_{s l z} T_{s l z}$, and $q_{c}=S_{w} k_{w}\left(T_{s l z}-T_{R}\right)+S_{b} k_{b}\left(T_{s l z}-T_{R}\right)$ into Eq. (1) yielded

$$
m_{s l z} c_{s l z} \frac{d T_{s l z}}{d t}=\tau \omega+\dot{m}_{i} c_{w} T_{w}-\dot{m}_{o} c_{s l z} T_{s l z}-S_{w} k_{w}\left(T_{s l z}-T_{R}\right)-S_{b} k_{b}\left(T_{s l z}-T_{R}\right)
$$

where, $c_{s / z}$ and $c_{w}$ are specific heat of the SLZ and the workpiece, respectively, $k_{w}$ and $k_{b}$ are thermal conductivity of the workpiece and the backing plate, respectively, and $S_{w}$ and $S_{b}$ are shape factors for a vertical cylinder in a semi-infinite medium and a disc on a semi-infinite medium, respectively [8]. $\dot{m}_{i}, S_{w}$ and $S_{b}$ are calculated from tool geometry, travel speed and tool rotation frequency. $q_{\text {gen }}$ is directly proportional to $m_{s / z}$, and $\omega$ was held constant in experiments, therefore,

$$
m_{s l z}=K \tau
$$

such that,

$$
K=\frac{m_{s l z-s}}{\tau_{s}}
$$

where, $m_{s l z-s}$ is mass of the SLZ and $\tau_{s}$ is torque, while at steady state. $m_{s l z-s}$ is calculated as per [9] and $\tau_{s}$ is determined later in this section (Eq.13).

To cast the equations into a more convenient form, the state variable, $x$ and input variable, $u$ are defined as

$$
\begin{aligned}
& x=\frac{T_{s l z}}{\tau} \\
& u=\frac{1}{\tau}
\end{aligned}
$$

Substituting Eqs. (2), (4), (6) and (7) into (3), and collecting constants together, 


$$
\frac{d x}{d t}=(C-B x+A u) u
$$

where, $A, B$ and $C$ are constants given by

$$
\begin{gathered}
A=\left(\frac{\dot{m}_{i} c_{s l z}+S_{s l z} k_{s l z}+S_{b} k_{b}}{K c_{s l z}}\right) T_{R} \\
B=\frac{\dot{m}_{i}}{K}+\frac{S_{s l z} k_{s l z}+S_{b} k_{b}}{K c_{s l z}} \\
C=\frac{\omega}{K c_{s l z}}
\end{gathered}
$$

$T_{s / z}$ is the process output given by

$$
T_{\text {slz }}=\frac{x}{u}
$$

Equations (8) and (12) represent the nonlinear thermal process model. Steady-state torque was given by Eqs. (6), (7) and (8) as

$$
\tau_{s}=\frac{B T_{S}-A}{C}
$$

\subsection{Defect model}

A reduction in the SLZ mass from its steady state value indicates the formation of a defect. Therefore, a disturbance model was established to estimate the SLZ mass deviation from steady-state, $\Delta m$. The ratio of the output, $T_{S L Z}$, and the state variable, $x$, from the process model gives the estimated torque. The difference in the estimated torque and the experimentally identified torque for good welds was related to the SLZ mass deviation from steady-state using Eq. (4). The disturbance model for $\Delta m[\mathrm{~kg}]$ is given by

$$
\Delta m=K\left(\frac{T_{s l z}}{x}-\tau\right)
$$

\subsection{Process/defect observer}

As described above, a reduction in SLZ mass indicates the formation of a defect. To estimate the SLZ mass deviation using the measured process output (i.e. tool-workpiece interface temperature) a disturbance observer has been designed using the process and disturbance models given in Eqs. (8) and (17), respectively. Figure 2 shows the schematic of the disturbance observer. A hat above the variables indicates that the values are model estimations. The operating principle of the observer is that the mathematical model of the process (Eq. 8) is simulated in parallel with the process. Since the model is not perfect in practice, it results in a difference between the real measured output ( $\left.T_{S L Z}\right)$ and estimated output $\left(\widehat{T_{S L Z}}\right)$ from simulation. This difference, known as error, is fed back to the process model through observer gain with the intent of driving the error term to zero. When the error term is close to zero (i.e. the process model output and measured process output are equal) we can assume that the estimated states $(\hat{x})$ and actual process states are also equal. In this case, the estimated states can then be used to calculate the disturbances/defects using Eq. (14). In addition to the observer gain, an integral term is introduced into the feedback path. Here, the error term is integrated with respect to time to eliminate small steady-state errors. 


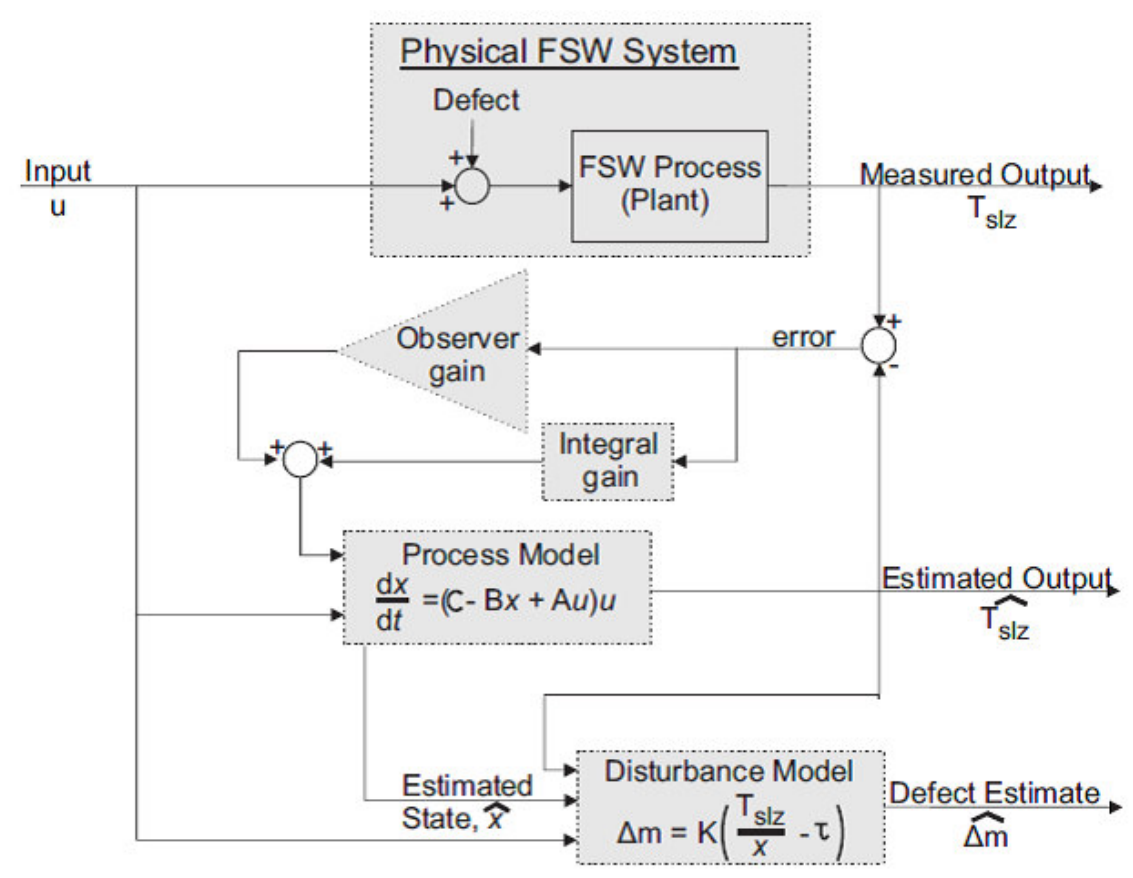

Figure 2. Schematic of the disturbance observer

\section{Materials and methods}

The experimental data used in this research was generated on a commercial 3-axis CNC mill. The mill is retrofitted with a current probe and a custom-built wireless tool-embedded thermocouple-based temperature measurement system [5], [6]. Friction stir welds were created with aluminum 6061-T6 and the specifics of the experimental setup, tool material and its dimensions, workpiece dimensions, and experimental results that were used for this work are given in [7].

\section{Results and discussion}

The disturbance observer described above was validated using a set of experimental FSW temperature measurements for aluminum 6061-T6 friction stir welds. Filtered experimental temperature data for welds with defects $(900 \mathrm{rpm}$ and $500 \mathrm{~mm} / \mathrm{min}$ ) and welds without defects $(1100 \mathrm{rpm}$ and $400 \mathrm{~mm} / \mathrm{min}$ ) were used as the measured output from the FSW process plant. In these experiments the process model input torque, $u$, was not directly measured but instead was inferred from tool rotation frequency and tool travel speed using an experimentally derived curve fit. Results for the SLZ mass deviation from steady-state, $\Delta m$ are shown in Fig. 3. A positive value of $\Delta m$ indicates a weld with no defects and negative value of $\Delta m$ indicates a weld in which defects are present. 

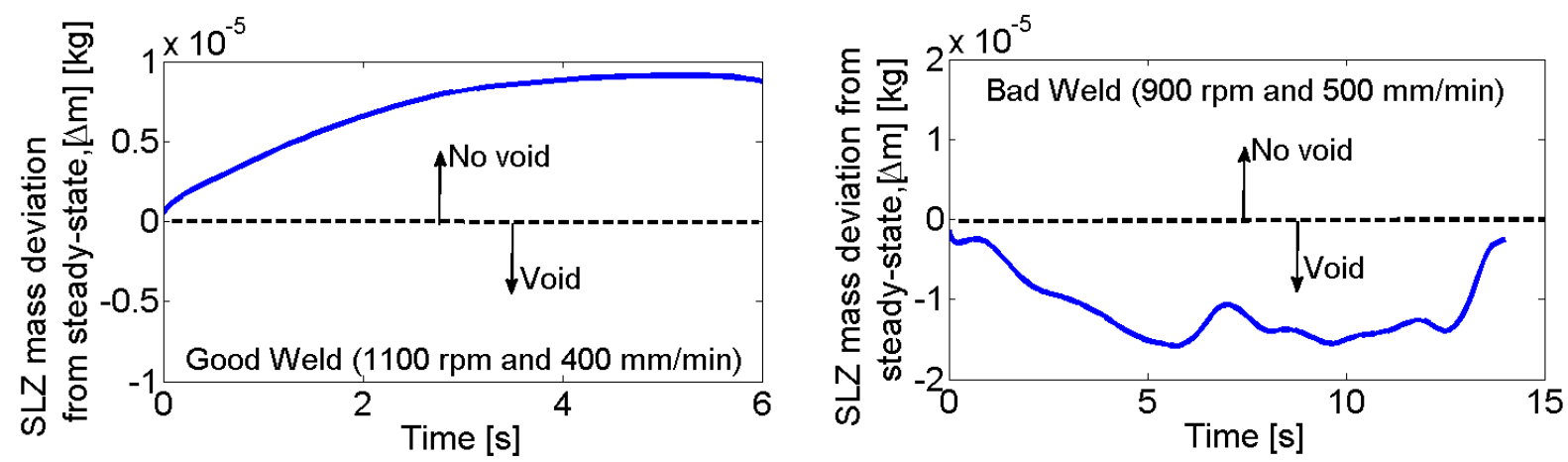

Figure 3. Plots of SLZ mass deviation from steady-state against time for welds with and without defects

Defects generated due to a poor combination of tool rpm and traverse speed can continue along the length of the weld (sometimes referred to as a wormhole). Since a wormhole is present along the whole weld length, the ratio of defect volume to weld volume is the same as the ratio of the cross-sectional defect area to the cross-sectional weld area (Fig. 4). Assuming a constant defect area throughout the length of the weld and constant density, the ratio of the SLZ mass deviation from steady-state, $\Delta \mathrm{m}$, to the steady-state SLZ mass, $m_{s l z-s}$, is same as the ratio of the defect cross-sectional area to the weld cross-sectional area. From a plot of the estimated SLZ mass deviation from steady-state, $\Delta m$, as a function of time for $900 \mathrm{rpm}$ and $500 \mathrm{~mm} / \mathrm{min}$ operating conditions it was determined that $\Delta m$ is approximately $12 \times 10^{-6} \mathrm{~kg}$, which is $1 / 50$ the steady-state SLZ mass (Fig. 4). The observed cross-sectional area of the defect in Fig. 4 was calculated to be 1/53 times the weld area using image processing software.

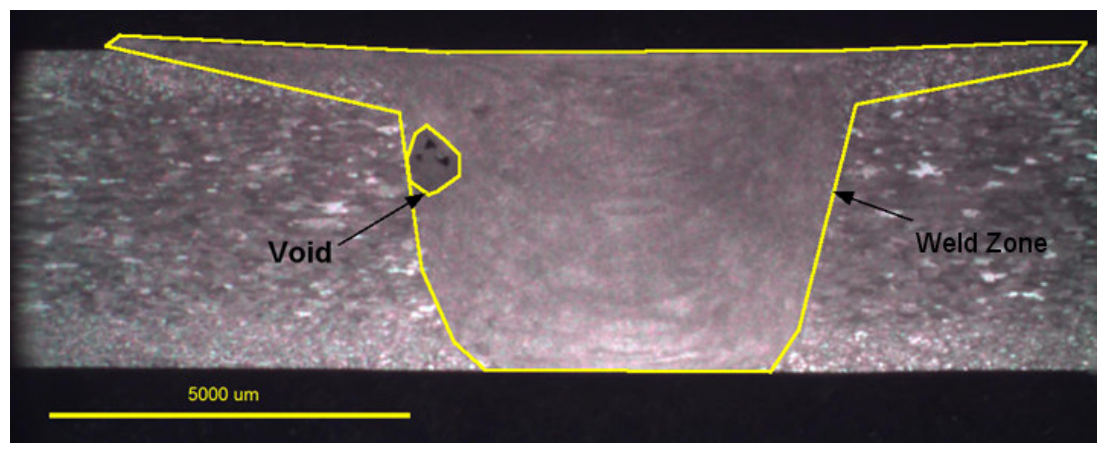

Figure 4. Macrograph of weld with sub-surface defect

\section{Conclusions}

A novel approach using a physics-based thermal model and experimental identification of the FSW process along with a preliminary disturbance model accounting for defects introduced by parameter variations were developed in this work. The approach has demonstrated to be able to differentiate between welds without defects and welds with subsurface defects that are due to input parameter variations.

The method of using tool-workpiece temperature signals with a disturbance-observer shows the potential for accurately detecting when sub-surface defects are created in a friction stir weld. Initial work shows that this method may be able to accurately predict the size of these defects, when formed. Further research on the quality of these welds, three-dimensional measurement of defects, and 
application to different alloys and weld geometries must be done to improve the method and gain confidence in the results.

\section{Acknowledgements}

The authors gratefully acknowledge the support of this work by National Science Foundation grant CMMI-1332738, the Wisconsin Alumni Research Foundation (WARF) Technology Development RA, the Innovation \& Economic Development Research (IEDR) Program, and the Machine Tool Technology Research Foundation.

\section{References}

[1] Thomas WM, Nicholas ED, Needham JC, Murch MG, Temple-Smith P, Dawes CJ. Improvements to Friction Welding.1995; Patent No. EP 0615480.

[2] Mishra RS, Ma ZY. Friction Stir Welding and Processing. Mater Sci Engineering 2005; R 50:1-78.

[3] Nishikawa H, Fujimoto M. Control of Rotational Distortion in Friction Stir Welding. Weld World 2004; 48:79-85.

[4] Mishra RS. Introduction. In: Mishra RS, Mahoney M, editors. Friction Stir Welding and Processing, Materials Park, OH: ASM International; 2007, p. 1-5.

[5] Fehrenbacher A. Enhancing Friction Stir Welding Through Process Instrumentation and Closed-Loop Control. PhD Thesis, University of Wisconsin-Madison 2012.

[6] Fehrenbacher A, Duffie NA, Ferrier NJ, Pfefferkorn FE, Zinn MR. Toward Automation of Friction Stir Welding Through Temperature Measurement and Closed-Loop Control. J Manufacturing Sci Engineering 2011; 133: 051008.1-051008.12.

[7] Fehrenbacher A, Duffie NA, Ferrier NJ, Pfefferkorn FE, Zinn MR. Effects of tool-workpiece interface temperature on weld quality and quality improvements through temperature control in friction stir welding. International J Advanced Manufacturing Technology 2014; 17:165-179.

[8] Incropera FP, Dewitt DP, Bergman TL, Lavine AS. Introduction to Heat Transfer. 5th ed. Wiley; 2006.

[9] Klevin D'Cunha. Predicting energy consumption during friction stir welding. MSME Thesis, University of Wisconsin-Madison 2011. 